\title{
Causas y consecuencias psicosociales del uso patológico de videojuegos en adolescentes
}

\author{
Fco. Javier López Fernández \\ frlopez@uji.es \\ Paula Etkin \\ etkin@uji.es \\ Jordi Ortet Walker \\ jortet@uji.es \\ Laura Mezquita Guillamón \\ Imezquit@uji.es \\ Manuel Ignacio Ibáñez Ribes \\ iribes@uji.es
}

\section{Resumen}

Los videojuegos son uno de los pasatiempos más populares. Una minoría de jugadores, especialmente entre los adolescentes, presentan sintomatología asociada al uso patológico o adicción. Por otra parte, el uso patológico de videojuegos se ha relacionado tanto a problemas externalizantes como internalizantes. Sin embargo, la escasez de estudios longitudinales nos impide conocer la dirección de estas asociaciones.

El objetivo del presente estudio es observar las relaciones causales entre el uso patológico de videojuegos y la sintomatología externalizante e internalizante en adolescentes.

Un total de 270 jugadores (193 varones), con una media de edad de 13,79 años $(D T=1,3)$ en tiempo 1 , fueron seguidos en las dos oleadas. Se evaluó el uso problemático de videojuegos y los problemas psicosociales. Para observar las causas y las consecuencias del uso patológico de videojuegos, se emplearon modelos de ecuación estructural autorregresivos mediante un diseño de panel de retardo cruzado de dos oleadas separadas por un año.

La ansiedad social predijo el uso patológico de videojuegos para ambos géneros, mientras que el uso patológico de videojuegos predijo la conducta antisocial en varones.

El presente estudio sugiere que los adolescentes con ansiedad social desarrollarían un uso patológico de videojuegos como método de afrontamiento de sus dificultades sociales. Así, en línea con esta idea, diversos estudios han mostrado que la baja competencia social es un factor de riesgo para el futuro desarrollo de uso problemático de videojuegos. 
Además, este uso patológico podría incrementar la conducta antisocial a largo plazo, deteriorando las relaciones interpersonales de los jugadores.

Palabras clave: adicción a los videojuegos, uso patológico de videojuegos, ansiedad social, conducta antisocial, adolescencia.

\section{Abstract}

Video games are one of the most popular pastimes. A minority of players, especially among adolescents, show symptomatology related to pathological gaming. On the other hand, pathological gaming has been associated with both externalizing and internalizing problems. Nevertheless, the lack of longitudinal studies prevents from exploring the direction of such relationships. The aim of the current study is to observe the causal relationships between pathological gaming and externalizing and internalizing symptomatology among adolescents.

A total of 270 players (193 males) with a mean age of 13.79 years $(S D=1.3)$ at wave 1 were followed one year later at wave 2. Pathological gaming and psychosocial problems were assessed. In order to know the causes and consequences of pathological gaming, a two-wave cross-lagged panel autoregressive model separated by 1 -year was employed.

Social anxiety predicted pathological gaming for both genders, whereas pathological gaming predicted antisocial behaviors among males.

The present study suggests that adolescents with social anxiety may develop a pathological video game use as a coping mechanism to overcome social problems. Thus, in line with this, some studies have found that social competence is a risk factor to develop future pathological gaming.

Furthermore, such pathological video gaming could increase long-term antisocial behavior, by disrupting players' interpersonal relationships.

Keywords: video game addiction, pathological gaming, social anxiety, antisocial behavior, adolescence.

\section{Introducción}

Actualmente los videojuegos son uno de los pasatiempos más populares. Así, 12 millones de personas juegan a videojuegos semanalmente en España, con una media de 6,6 horas por semana; la mayor frecuencia de uso se encuentra en población infantil y adolescente (Asociación Española de Videojuegos 2017).

A pesar de que la mayoría de jugadores no tienen problemas relacionados con el uso de videojuegos, una minoría de estos, especialmente entre los adolescentes (Mihara y Higuchi 2017), presentan sintomatología asociada al uso patológico o adicción, como tolerancia, saliencia, modificación del estado de ánimo, abstinencia (psicológica), conflicto y recaída (Griffiths 2005; Petry, Rehbein, Ko, y O’Brien 2015). En este sentido, tanto la American Psychiatric Association, en la sección de trastornos que requieren mayor estudio (American Psychiatric Association 2013, 795), como la Organización Mundial de la Salud, incluyen la adicción a los videojuegos como trastorno mental (World Health Organization 2018). Particularmente en España, alrededor del $8 \%$ de los adolescentes ha sido categorizado como jugador patológico 
(Buiza-Aguado, Alonso-Canovas, Conde-Mateos, Buiza-Navarrete y Gentile 2018; Lopez-Fernandez, Honrubia-Serrano, Baguley y Griffiths 2014).

Este uso patológico de videojuegos se ha asociado en adolescentes tanto a problemas externalizantes, por ejemplo, hiperactividad o conducta antinormativa, como internalizantes, por ejemplo, problemas emocionales como ansiedad o depresión (Dreier et al. 2017; Müller et al. 2015; Wartberg et al. 2017). Sin embargo, la escasez de estudios longitudinales no nos permite conocer adecuadamente la dirección causal de estas asociaciones.

Una manera de superar esta limitación es el empleo de ecuaciones estructurales mediante modelos autorregresivos, una herramienta ya utilizada en el estudio de las causas y consecuencias entre el uso patológico de videojuegos y constructos psicosociales (Lemmens, Valkenburg y Peter 2011). Los modelos autorregresivos eliminan una considerable proporción de la varianza de terceras variables e incrementan la validez de la influencia de los predictores en tiempo 1 sobre las variables dependientes en tiempo 2 (Schlüter, Davidov y Schmidt 2007).

Así, el objetivo del presente trabajo es estudiar las relaciones causales entre el uso patológico de videojuegos y la sintomatología externalizante e internalizante en adolescentes, mediante un diseño de panel de dos oleadas separadas por un año.

\section{Método}

\section{Participantes y procedimiento}

El estudio formó parte de una investigación más amplia sobre factores psicosociales involucrados en la salud mental adolescente. Así, los participantes fueron estudiantes de institutos de secundaria españoles. Los cuestionarios fueron voluntariamente completados por aquellos estudiantes autorizados por sus tutores legales. De la muestra inicial de 776 adolescentes en año 1, se seleccionó a un total de 270 jugadores (193 varones) que jugaban al menos alguna vez por semana en la primera oleada y completaron los cuestionarios de la segunda oleada un año después. Así, en la primera oleada, la media de edad fue de 13,79 años (DT=1,3) con un rango de edad entre 12 y 17 años.

\section{Instrumentos}

Se evaluó el uso problemático de videojuegos mediante una adaptación de una escala para adolescentes (Gentile 2009). La escala se compone de 11 ítems que evalúan la frecuencia de problemas relacionados con videojuegos en una escala Likert de 4 puntos (desde $0=$ nunca o casi nunca hasta $3=$ siempre o casi siempre). El alfa de Cronbach fue de 0,88 .

Para evaluar problemas psicosociales en la adolescencia, se empleó la escala SENA, Sistema de Evaluación de Niños y Adolescentes (Fernández-Pinto, Santamaría, Sánchez-Sánchez, Carrasco y del Barrio 2015), que identifica un amplio espectro de problemas sociales y conductuales en individuos de 3 a 18 años. Se utilizaron 114 ítems para evaluar sintomatología en problemas externalizantes (agresividad, problemas en el control de la ira, conducta antisocial, conducta desafiante, problemas atencionales e hiperactividad) e internalizantes (depresión, ansiedad, ansiedad social, quejas somáticas y sintomatología postraumática). La escala está compuesta por 5 opciones de respuesta, desde 0 (nunca o casi nunca) a 4 (siempre o casi siempre). 
Análisis de datos

Para estudiar las causas y las consecuencias entre estas variables, se emplearon modelos de ecuación estructural con un diseño de panel de retardo cruzado autorregresivo (Cross-lagged panel autoregressive model), mediante el empleo del programa AMOS 7.0.

\section{Resultados}

El 6,4 \% de la muestra inicial de adolescentes, de donde se extrajo la submuestra de jugadores, fue categorizado como jugador patológico acorde a los criterios de la escala (Gentile 2009). La ansiedad social predijo el uso patológico de videojuegos en ambos géneros (véase la figura 1), mientras que el uso patológico predijo la conducta antisocial en varones (véase la figura 2). Por otra parte, tanto el uso patológico como las variables psicosociales presentaron una alta estabilidad temporal un año después. No se encontraron más asociaciones significativas temporales del uso patológico de videojuegos con el resto de sintomatología internalizante y externalizante.

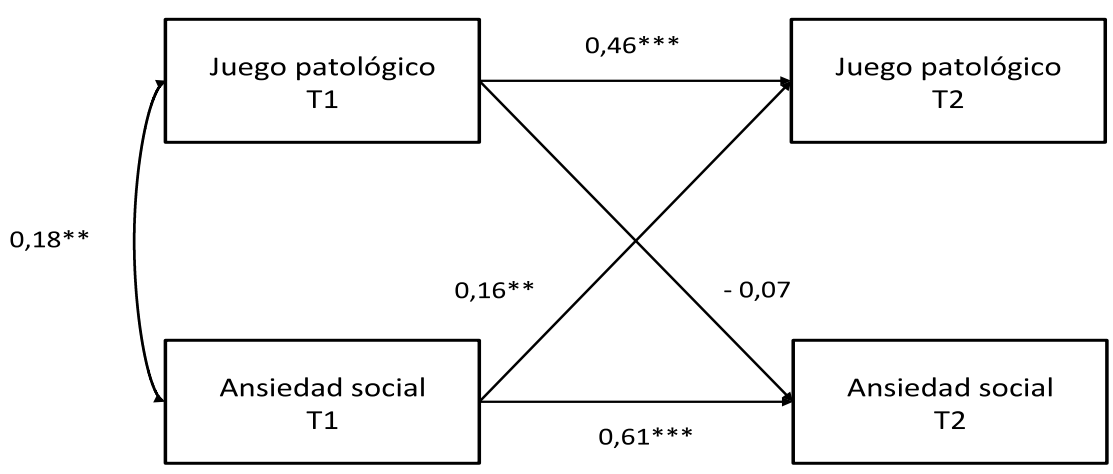

Figura 1. Modelo autorregresivo de la relación causal entre juego patológico $y$ ansiedad social. ${ }^{*} p<0,05 ;{ }^{* *} p<0,01 ;{ }^{* *} p<0,001$. 


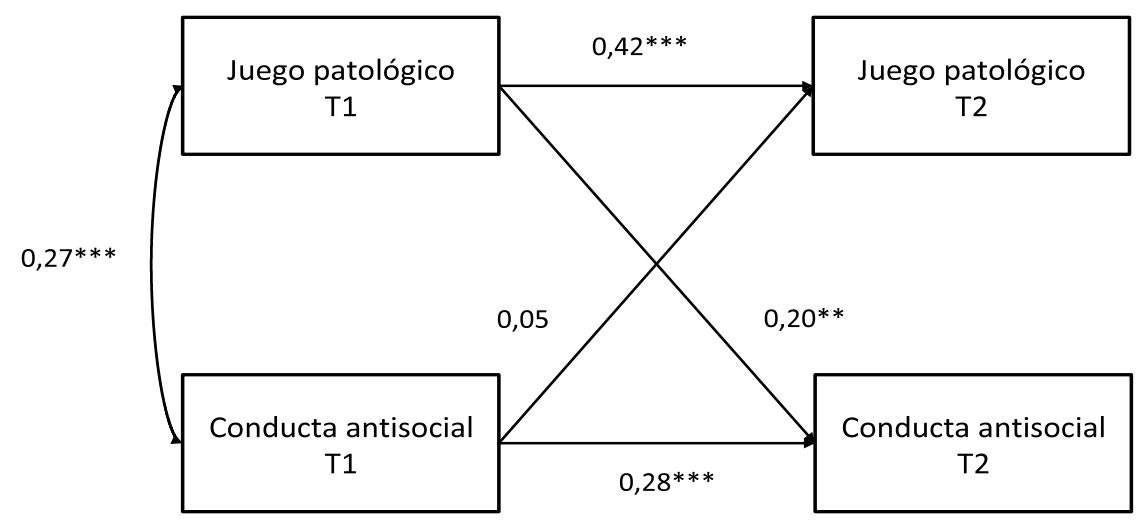

Figura 2. Modelo autorregresivo de la relación causal entre juego patológico y conducta antisocial en varones. ${ }^{*} p<0,05 ;{ }^{* *} p<0,01 ;{ }^{* * *} p<0,001$.

\section{Discusión y conclusiones}

El presente estudio examinó longitudinalmente las causas y las consecuencias en las relaciones entre problemas psicosociales y el uso patológico de videojuegos en adolescentes. Los resultados sugieren que los adolescentes con ansiedad social desarrollarán un uso patológico de videojuegos como método de afrontamiento a sus dificultades sociales. Así, acorde a esto, diversos estudios han mostrado que la baja competencia social es un factor de riesgo para el futuro desarrollo del uso patológico de videojuegos (Gentile et al. 2011; Lemmens, Valkenburg y Peter 2011). En esta línea, variables como pobres relaciones interpersonales (Kim, Namkoong, Ku y Kim 2008) o miedo a las relaciones sociales y a la evaluación negativa (Škařupová y Blinka 2015) han sido relacionadas con la adicción a los videojuegos. En este sentido, los individuos tímidos deben usar los videojuegos para enfrentarse a dificultades sociales en la vida real, con el objetivo de generar amistades online (Kowert, Domahidi y Quandt 2014). Además, este uso patológico en chicos favorecería la expresión de comportamientos antinormativos y antisociales a largo plazo. La muestra total en año 1 mostró una prevalencia de uso problemático de videojuegos del $6,4 \%$. Este dato probablemente esté ligeramente subestimado, puesto que la escala empleada para la evaluación del uso problemático de videojuegos fue desarrollada antes de la propuesta del trastorno en el DSM-5. Así, mientras que el DSM-5 establece un mínimo de 5 criterios para el diagnóstico, la escala utilizada establece 6. Por lo tanto, según este y otros estudios, podemos decir que la prevalencia de uso problemático de videojuegos en adolescentes españoles se encuentra entre el 7 y el $8 \%$ (Buiza-Aguado, AlonsoCanovas, Conde-Mateos, Buiza-Navarrete y Gentile 2018; Lopez-Fernandez, Honrubia-Serrano, Baguley y 2014).

Este estudio no encontró más asociaciones longitudinales entre el uso problemático de videojuegos y el extenso espectro de sintomatología psicológica al que fue examinado. 
De esta manera, el resto de asociaciones observadas en la literatura en estudios de diseños transversales entre el uso problemático de videojuegos y problemas psicológicos se debería a la influencia de terceras variables como rasgos de personalidad (por ejemplo, neuroticismo o impulsividad).

Se ha de destacar que todos los datos fueron extraídos de autoinformes susceptibles a sesgos como la deseabilidad social. Futuros estudios deberían comprobar la relación causal del uso patológico de videojuegos con sintomatología externalizante e internalizante en muestras más amplias y representativas, además de emplear un mayor número de oleadas para poder ver la evolución de estas asociaciones, mediante modelos de curva latente.

Este estudio muestra que la prevención y el tratamiento del uso patológico de videojuegos en adolescentes debería centrarse potencialmente en estimular actividades interpersonales para el desarrollo de habilidades sociales, incrementando así contactos sociales fuera de los videojuegos.

En conclusión, el presente estudio muestra que las dificultades sociales de los jugadores son un factor de riesgo para el futuro desarrollo de uso patológico de videojuegos, algo frecuentemente encontrado en la investigación. Además, sugiere que en chicos este uso patológico podría potenciar conductas antisociales.

\section{Referencias bibliográficas}

American Psychiatric Association. 2013. Diagnostic and statistical manual of mental disorders (5th ed.). Arlington, VA: American Psychiatric Publishing.

Asociación Española de Videojuegos (AEVI). 2017. El anuario del videojuego 2017. Recuperado de http://www.aevi.org.es/documentacion/el-anuario-delvideojuego/ [Consultado el 29 de junio de 2018].

Buiza-Aguado, Carlos, Araceli Alonso-Canovas, Celia Conde-Mateos, Juan Jose Buiza-Navarrete y Douglas Gentile. 2018. «Problematic video gaming in a young Spanish population: association with psychosocial health». Cyberpsychology, Behavior, and Social Networking, 21(6): 388-394.

Dreier, Michael, Klaus Wölfling, Eva Duven, Sebastián Giralt, Manfred E. Beutel y Kai W. Müller. 2017. «Free-to-play: about addicted Whales, at risk Dolphins and healthy Minnows. Monetarization design and internet gaming disorder». Addictive behaviors, 64: 328-333.

Fernández-Pinto, Irene, Pablo Santamaría, Fernando Sánchez-Sánchez, Miguel Ángel Carrasco y Victoria del Barrio. 2015. SENA -Sistema de evaluación de niños y adolescentes. Manuel de aplicación, corrección e interpretación-. Madrid: TEA.

Gentile, Douglas. 2009. "Pathological video-game use among youth ages 8 to 18: a national study». Psychological Science, 20(5): 594-602.

Gentile, Douglas, Hyekyung Choo, Albert Liau, Timothy Sim, Dongdong Li, Daniel Fung y Angeline Khoo. 2011. "Pathological video game use among youths: a two-year longitudinal study». Pediatrics, 127(2): e319-e329.

Griffiths, Mark. D. 2005. «A 'components' model of addiction within a biopsychosocial framework». Journal of Substance Use, 10: 191-197.

Kim, Eun Joo, Kee Namkoong, Taeyun Ku y See Joo Kim. 2008. "The relationship between online game addiction and aggression, self-control and narcissistic personality traits». European psychiatry, 23(3): 212-218.

Kowert, Rachel, Emese Domahidi y Thorsten Quandt. 2014. «The relationship between online video game involvement and gaming-related friendships among 
emotionally sensitive individuals». Cyberpsychology, Behavior, and Social Networking, 17(7): 447-453.

Lemmens, Jeroen S., Patti M. Valkenburg y Jochen Peter. 2011. «Psychosocial causes and consequences of pathological gaming». Computers in Human Behavior, 27(1): 144-152.

Lopez-Fernandez, Olatz, Maria Luisa Honrubia-Serrano, Thom Baguley y Mark D. Griffiths. 2014. "Pathological video game playing in Spanish and British adolescents: Towards the exploration of Internet Gaming Disorder symptomatology». Computers in Human Behavior, 41: 304-312.

Mihara, Satoko y Sumusu Higuchi. 2017. «Cross-sectional and longitudinal epidemiological studies of internet gaming disorder: A systematic review of the literature». Psychiatry and Clinical Neurosciences, 71(7): 425-444.

Müller, Kai W., Mari Janikian, M. Dreier, Klaus Wölfling, Manfred Beutel, Chara K. Tzavara, Clive Richardson y A. Tsitsika. 2015. «Regular gaming behavior and internet gaming disorder in European adolescents: results from a cross-national representative survey of prevalence, predictors, and psychopathological correlates». European Child \& Adolescent Psychiatry, 24(5): 565-574.

Petry, Nancy M., Florian Rehbein, Chih-Hung Ko y Charles P. O'Brien. 2015. «Internet gaming disorder in the DSM-5». Current psychiatry reports, 17(9): 72.

Schlüter, Elmar, Eldad Davidov y Peter Schmidt. 2007. «Applying autoregressive cross-lagged and latent growth curve models to a three-wave panel study». En Longitudinal models in the behavioral and related sciences, ed. Kee Van Monfort, Johan Oud y Albert Satorra. London / New York: Routledge.

Škařupová, Kateřina y Lukas Blinka. 2015. «Interpersonal dependency and online gaming addiction». Journal of behavioral addictions, 5(1): 108-114.

Wartberg, Lutz, Levente Kriston, Michaela Kramer, Anja Schwedler, Tania M. Lincoln y Rudolf Kammerl. 2017. "Internet gaming disorder in early adolescence: Associations with parental and adolescent mental health». European Psychiatry, 43: 14-18.

World Health Organization. 2018. International statistical classification of diseases and related health problems (11th Revision). Recuperado de https://icd.who.int/browse11//-m/en [Consultado el 5 de junio de 2018]. 